\title{
GRASS: A Gradient-Based Random Sampling Scheme for Milano Retinex
}

\author{
Michela Lecca, Alessandro Rizzi, and Raul Paolo Serapioni
}

\begin{abstract}
Retinex is an early and famous theory attempting to estimate the human color sensation derived from an observed scene. When applied to a digital image, the original implementation of retinex estimates the color sensation by modifying the pixels channel intensities with respect to a local reference white, selected from a set of random paths. The spatial search of the local reference white influences the final estimation. The recent algorithm energy-driven termite retinex (ETR), as well as its predecessor termite retinex, has introduced a new path-based image aware sampling scheme, where the paths depend on local visual properties of the input image. Precisely, the ETR paths transit over pixels with high gradient magnitude that have been proved to be important for the formation of color sensation. Such a sampling method enables the visit of image portions effectively relevant to the estimation of the color sensation, while it reduces the analysis of pixels with less essential and/or redundant data, i.e., the flat image regions. While the ETR sampling scheme is very efficacious in detecting image pixels salient for the color sensation, its computational complexity can be a limit. In this paper, we present a novel Gradient-based RAndom Sampling Scheme that inherits from ETR the image aware sampling principles, but has a lower computational complexity, while similar performance. Moreover, the new sampling scheme can be interpreted both as a path-based scanning and a 2D sampling.
\end{abstract}

Index Terms-Path-based Retinex model, spatial color correction, image enhancement.

\section{INTRODUCTION}

$\mathbf{T}$ HE human color sensation deriving from the observation of a certain point of a scene may differ from the physical color signal coming from that point. In fact, several experiments revealed that the color sensation at a point depends not only on the photometric properties of that point, but also on the physical cues and spatial arrangement of the colors surrounding that point [1]-[3].

Retinex [4] is an early computational model that attempts to estimate the color sensation. Retinex model follows the human vision structure for which the color signal is processed initially by the retina photoreceptors and then by the cortex performing

Manuscript received March 15, 2016; revised August 2, 2016 and December 12, 2016; accepted March 16, 2017. Date of publication March 23, 2017; date of current version April 18, 2017. This work was carried out by the Research in Pairs Program of the Center for Mathematics Research of the Fondazione Bruno Kessler, Trento, Italy, in collaboration with different research centers. The associate editor coordinating the review of this manuscript and approving it for publication was Dr. Keigo Hirakawa. (Corresponding author: Michela Lecca.)

M. Lecca is with Fondazione Bruno Kessler, Center for Information and Communication Technology, 38123 Trento, Italy (e-mail: lecca@fbk.eu).

A. Rizzi is with the Dipartimento di Informatica, Università degli Studi di Milano, 20135 Milano, Italy (e-mail: alessandro.rizzi@unimi.it).

R. P. Serapioni is with the Dipartimento di Matematica, Università degli Studi di Trento, 38123 Trento, Italy (e-mail: raulpaolo.serapioni@unitn.it).

Color versions of one or more of the figures in this paper are available online at http://ieeexplore.ieee.org.

Digital Object Identifier 10.1109/TIP.2017.2686652 a spatial interaction among the colors of the observed scene. Across this pipeline, the chromatic components of the color signal are processed independently. When applied to a noncalibrated digital color picture, Retinex produces an enhanced color image, where the chromatic dominant of the light and possible smooth shadows are lowered, while scene details and edges are enhanced. This corresponds to a qualitative estimation of the color sensation [5]. In order to consider the mutual influence of adjacent colors in an image, Retinex performs a spatial exploration of each pixel neighborhood. Then it modifies the pixel color with respect to a local reference white, selected from the explored region. Many implementations of Retinex are available in the literature [6]. They differ to each other in the way to spatially explore the neighborhood of each pixel and in the way to process adjacent colors. The original Retinex implementation scans the neighborhood of an image pixel $x$ by a set of random paths ending in $x$. The chromatic intensities of the color sensation at $x$ are obtained as the averages, among these random paths, of the relative ratios of the channel intensities of subsequent pixels along each path, and division by zero is prevented.

The path-based scanning approach of the original Retinex has been adopted by many other subsequent Retinex implementations, e.g. [7]-[9].

The work we present here originates from two recent path-based implementations of Milano Retinex [3], [10], a class of Retinex algorithms mainly developed for color image enhancement: Termite Retinex (TR) [11] and Energy-driven Termite Retinex (ETR) [12]. The main novelty of these methods is their image aware spatial color sampling, i.e. their sampling procedure takes into account visual cues of the image. Precisely, in TR and ETR, the Retinex paths are thought as the traces of a swarm of artificial termites traveling from any pixel $x$ in search for a local white reference. Each termite path depends on the image edges, on the spatial position of the termite, and on the routes previously traveled by other termites (if any). The dependence on the edges is justified by the important role that edges play in the formation of the color sensation [3], [13]-[15]. The dependence on the position has been introduced to push the termite route over the image in search of a local reference white. Finally, the dependence on the geometry of the paths previously traveled is inspired by an adaptation of the stigmergic behaviour of a natural swarm of termites foraging for food: it allows to optimize the spatial image exploration by discouraging the multiple visit of image regions already considered.

As Retinex, TR and ETR process separately the three chromatic channels of the input image. In TR, any termite starts from a pixel $x$, and it chooses its next step by maximizing 
the local contrast over a $3 \times 3$ window, within the constraints imposed by stigmergy and distance. In ETR, any termite route is computed as the local minimum of an energy functional, designed so as to favor the visit of sharp edges (i.e. pixels with high gradient magnitude) that are close to $x$, according to the evidence that the chromatic influence among adjacent colored regions is related to their relative position [3], [16]. In this functional, stigmergy is modeled by a term that discourages the over-exploration of already visited image portions.

The edge based sampling schemes of TR and ETR provide an efficient way to spatially explore the pixel neighborhood in search for a local white reference: they favor the analysis of image pixels containing information relevant to color sensation, while they disadvantage the collection and processing of data coming from less important image areas. The sampling scheme of ETR is particularly efficacious in this respect, however its practical usage is adversely affected by its high computational complexity mainly due to the minimization of the energy functional.

TR and ETR have paved the way for the development of a new class of Retinex implementations, characterized by image aware sampling schemes. This topic is a challenging and attractive issue, both from a psycophysical and algorithmic point of view. In fact, it addresses the problem to define a set of visual features that possibly influence the color sensation and to include them in a novel Retinex model in an efficient way. As mentioned above, this research direction is still poorly investigated.

This paper aims at enriching the current state of the art in the image aware spatial sampling. Specifically, here we propose GRASS, a novel Gradient-based RAndom Sampling Scheme for Milano Retinex [3], [10], which is based on the same features of ETR (e.g. stigmergy, image gradient, spatial distance), but is characterized by a much lower computational complexity. Basically, GRASS works as follows. For each chromatic channel, GRASS explores the neighborhood of a pixel $x$ by $N$ random squares, having different size and orientation (see Figure 1 for an example), scans each square exhaustively by parallel segments, and detects over each segment a pixel maximizing the gradient magnitude weighted by a function of the pixel distance from $x$. Then GRASS processes the neighborhood of the pixels selected on the squares in order to find out a local white reference, and computes the final color sensation at $x$ in the same way as in ETR.

The exploration mechanism of GRASS is similar to that of a flat scanner. Each square can be viewed as the document plane of the scanner, while each scanning segment is like the moving linear sensor at a fixed time. In this framework, any pixel selected by GRASS in the square is what the linear sensor detects over the scanned segment and saves to build up the final digital picture.

GRASS entails two main novelties. First, its sampling figure at any image pixel $x$ (i.e. the set of pixels sampled around $x$ ) is similar to that output by ETR, in the sense that both ETR and GRASS favor the sampling of the image pixels, but the GRASS computation has a much lower complexity than that of ETR. This makes GRASS much more practicable than ETR. Second, GRASS has an interesting characteristic: its sampling

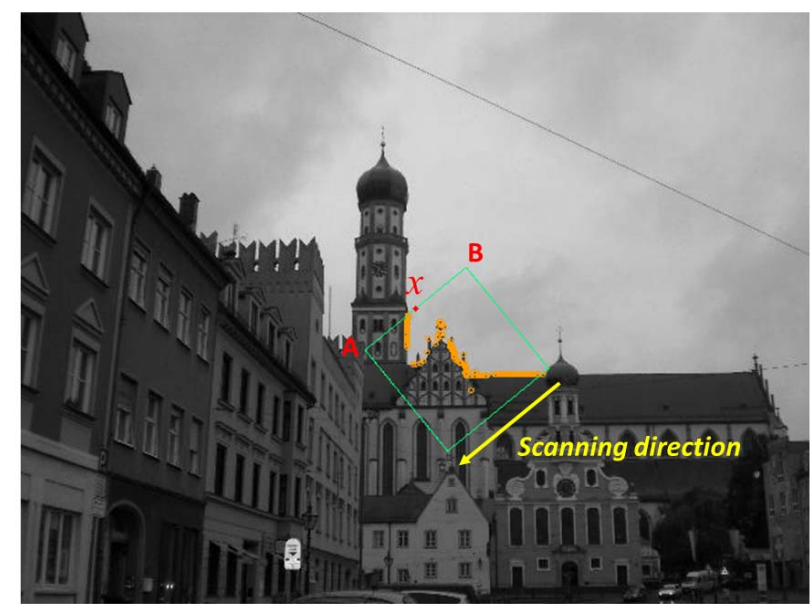

Fig. 1. GRASS scans the neighborhood of any image pixel $x$ by random squares. This figure shows such a square (in green) for the barycenter $x$ (in red) of the image support. The algorithm scans the segments parallel to the side $A B$; the orange circles highlight the pixels selected along each segment. See Section III for more details.

scheme can be regarded both as a path-based approach and as a bi-dimensional spatial sampling as well. Precisely, the interpretation of GRASS as a path-based sampling scheme arises when we look at the pixels sampled from each square as at the control points of a curve scanning the square. At the same time, since we do not impose any constraint about the connectivity between the square pixels and de facto we do not compute any path, GRASS can be interpreted as 2D sampling of the gradient magnitude map in a neighborhood of $x$. Due to this characteristic, GRASS can be also viewed as evolution of the 2D spatial sampling proposed by Random Spray Retinex (RSR) [17], where the figure sampling of any pixel $x$ is a set of pixels distributed around $x$ with radial density. However, we highlight that GRASS strongly differs from RSR and from its further versions STRESS [18] and QBRIX [19], because these methods do not take into account the image content for sampling.

The paper is organized as follows: Section II introduces some notation used in the paper, and describes the algorithms TR, ETR, RSR, STRESS and QBRIX; Section III explains the mathematical and technical details of GRASS; Section IV reports the experiments measuring the GRASS performance on image enhancement; finally, Section V contains our conclusions.

\section{RELATED WORK}

This Section is devoted to the description of related work. In Subsection II-A we introduce some notation used throughout the paper. In Subsection II-B we describe the algorithms TR and ETR, that first propose an image aware image sampling and that can be thus considered the predecessors of GRASS. In Subsection II-C we briefly describe RSR and its further versions STRESS and QBRIX.

\section{A. Notation}

Let us introduce some notation, that will be used in the following. 
We denote a chromatic channel of an RGB image $J$ by $I$. In this framework, $I$ is described as a function $I: \operatorname{Supp}(I) \rightarrow$ $[0,1]$, where $\operatorname{Supp}(I)$ is the set of the spatial coordinates of the image pixels. We indicate the number of pixels of $I$ by $|I|$ and we use $x$ to indicate a pixel in $\operatorname{Supp}(I)$.

We indicate the chromatic component of the color sensation deriving from $I$ by $S$ and we refer to $S$ also as the filtered version of $I . S$ is an 1D image, defined as a function from $\operatorname{Supp}(I)$ to $[0,1]$. The color sensation of a color image $J$ is a RGB image defined over $\operatorname{Supp}(J)$ and its chromatic components are the filtered versions of the chromatic components of $J$.

For all the methods described next, in order to prevent division by zero, any intensity value $I(x)=0$ is replaced with $I(x)=\varepsilon$, with $\varepsilon$ close to zero. In particular, in the experiments presented here $\varepsilon=10^{-} 6$.

Finally, we use the notation $\|\cdot\|$ to indicate the Euclidean norm of the vector ".".

\section{B. Image Aware Image Sampling: TR and ETR}

Termite Retinex (TR) explores the neighborhood of any pixel $x$ of $I$ by means of $N$ paths $\gamma_{1}, \ldots, \gamma_{N}$ with length $l$, viewed as the traces of $N$ termites $T_{1}, \ldots, T_{N}$.

For each $i=1, \ldots, N$, the path $\gamma_{i}$ is represented as an ordered set of pixels $x_{1}, \ldots, x_{l}$, where $x_{1}:=x$. For each $k=2, \ldots, l$, the pixel $x_{k}$ is chosen in the $3 \times 3$ window $N\left(x_{k-1}\right)$ centered at $x_{k-1}$, so that $x_{k}$ maximizes the function $f_{k}: N\left(x_{k-1}\right) \rightarrow[0,+\infty)$ defined as:

$$
f_{k}(y)=\frac{1}{K}[\theta(y)]^{\alpha}\left[\frac{\left\|x_{k-1}-y\right\|+\left|I\left(x_{k-1}\right)-I(y)\right|}{\sqrt{2}}\right]^{\beta}
$$

over the set of pixels in $N\left(x_{k-1}\right)$ never traveled by $T_{i}$, i.e. $x_{k} \neq x_{h}$ for any $h<k$.

The function $\theta$ is named the poison term and implements the stigmergy: it penalizes the value of $f_{k}$ on each pixel already traveled by a termite. Precisely, for each $x, \theta$ is initialized to 1.0 , then the value $\theta$ at a pixel $y$ is decreased of a fixed positive quantity $\delta$ each time a termite passes over $y$. The parameters $\alpha$ and $\beta$ weight the contribution of the poison to the term including the spatial distance and the local contrast (in particular, $\alpha, \beta \geq 0$ and $\alpha+\beta=1$ ). When the function $f_{k}$ is constant over the pixels of $N\left(x_{k-1}\right)$ not yet traveled by the termite, the termite chooses randomly its next step. The denominator $K$ is a normalization term introduced to rescale $f_{k}(y)$ in $[0,1]$ and to interpret it as a probability density function. The parameters $N, l, \delta, \alpha, \beta$ are user inputs.

The computational complexity of the TR sampling approach is $\mathcal{O}(N l|I|)$. Based on some experimental results, the work in [11] suggests to set up $l$ as the $70 \%$ of the length of the diagonal of $\operatorname{Supp}(I)$. This makes the complexity of TR much smaller than $\mathcal{O}\left(N|I|^{2}\right)$.

After the image exploration, TR computes the color sensation by the ratio-product-reset mechanism of MI-Retinex [3], as described in [7] and reported in [11].

The sampling scheme of TR has been proved to be efficacious for image exploration. However, some inefficiency still remains where a termite transits over a flat region. In this case, the termite selects randomly its next step, therefore it may happen that the termite scans the entire flat region, collecting redundant and unessential information for color sensation.

Energy-driven Termite Retinex (ETR) solved this problem by imposing global mathematical conditions to the paths. In order to manage the concept of functional minimization in a straightforward manner, in [12] the ETR scheme was formalized over a continuous domain. In this framework, ETR explores the neighborhood of any pixel $x$ by $N$ self-avoiding, continuous paths $\gamma_{1}, \ldots, \gamma_{N}:[0,1] \rightarrow \mathbf{R}^{\mathbf{2}}$ which, as in TR, are computed by an iterative procedure.

For each $i=1, \ldots, N$, the path $\gamma_{i}$ connects $\gamma_{i}(0):=x$ to another pixel $\gamma_{i}(1):=y_{i}$, that is sampled randomly from a uniform distribution defined over $\operatorname{Supp}(I)$ and $y_{i} \neq y_{j}$ for any $j \neq i, j=1, \ldots, N$. Moreover, $\gamma_{i}$ minimizes the energy functional

$E_{x}(\gamma)=\int_{0}^{1}\left[\frac{1}{1+d(x, \gamma(s))\|\nabla I(\gamma(s))\|^{2}}+\theta_{x}(\gamma(s))\right] d s$

over the set of continuous paths from $[0,1]$ to $\mathbf{R}^{\mathbf{2}}$ scanning $\operatorname{Supp}(I)$, where $\nabla I$ indicates the gradient of $I$ and $d$ is a spatial distance term defined as

$$
d(x, \gamma(s))=D^{2}-\|x-\gamma(s)\|^{2}
$$

and $D$ is the length of the diagonal of $\operatorname{Supp}(I)$.

The function $\theta_{x}: \operatorname{Supp}(I) \rightarrow[0,+\infty)$ is a poison term, analog to that of ETR. $\theta_{x}$ is initialized to zero when $i=1$. Then, for any $i>1$, the value of $\theta_{x}$ is increased by a quantity $\delta_{x}$ over the pixels traveled by the termite $T_{i-1}$. $N$ and $\delta_{x}$ are user input, but Lecca et al. [12] also suggest to set up them according to the image visual content.

The color sensation at $x$ is then computed as proposed in [17] by the following equation:

$$
S(x)=\frac{1}{N} \sum_{i=1}^{N} \frac{I(x)}{\max \left\{I\left(\gamma_{i}(t)\right): t \in[0,1]\right\}} .
$$

The sampling scheme of ETR is very efficacious: the termites poorly visit uniform regions, while cover more the image portions close to the nest $x$ and containing edges. Nevertheless, in practice, the usage of ETR is adversely affected by its computational burden, mainly due to the minimization procedure: the computational complexity of ETR is $\mathcal{O}\left(N|I|^{2} \log (|I|)\right)$.

\section{2D Spatial Sampling: RSR, STRESS and QBRIX}

The algorithm RSR [17] replaces the path-based approach of the original Retinex implementation with a 2D spatial sampling. Precisely, RSR scans the image regions around each pixel $x$ by a number of random sprays. A random spray is a set of pixels randomly selected from a circular neighborhood of $x$ with a radial density. Each chromatic value of the output at $x$ is then computed as the mean value of the ratios between the intensity at $x$ and the maximum intensity of each spray, averaged over the number of sprays, and division by zero is prevented.

STRESS [18] (Spatial-Temporal Retinex-inspired Envelope with Stochastic Sampling) is a variant of RSR, particularly 
suitable for local contrast stretching, automatic color correction, spatial color gamut mapping, and efficient color to gray-scale conversion, e.g. [20], [21]. As RSR, STRESS explores the neighborhood of each image pixel by random sprays, but it uses a different equation to estimate the pixel output. For each chromatic channel, and for each image pixel $x$, STRESS computes the lightest and the darkest pixel in each spray centered at $x$ and uses these values to define two envelope functions that contain the chromatic signal. The red, green and blue chromatic intensities of $x$ are thus stretched between the corresponding minimum and maximum values in the envelopes.

QBRIX [19] (Quantile-Based approach to RetIneX) is a probabilistic formulation of RSR. It removes the sampling procedure completely and models the spatial arrangement of the colors around any image pixel through a suitable probabilistic distribution function (pdf). QBRIX relies on two main observations: first, colors rarely occurring in the image are irrelevant to final color filtering; second, as already pointed out by RSR, the chromatic influence of the colors of the pixels around a pixel $x$ decreases by increasing the distance from $x$. These observations lead to two different implementations. The first one is a global filter (here denoted by G-QBRIX). The pdf of each chromatic channel is computed and the intensity value corresponding to a quantile fixed by the user, is set up as reference white. The color filtering is obtained by rescaling the chromatic values of the image by the selected quantile which controls the percentage of colors to be discarded. The second implementation takes into account also the second observation and leads to a local filter (here denoted by L-QBRIX). For each chromatic channel and for each pixel $x$, the algorithm computes a pdf at $x$, where the contribution of each image pixel is weighted by its distance from $x$. The color filtering at $x$ is then computed as in G-QBRIX. Disregarding the random sampling, QBRIX computes a final filtered image with a negligible chromatic noise. Since GRASS also considers the spatial distance, in the comparative analysis presented next, we considered L-QBRIX only.

\section{GRASS}

The Gradient-based RAndom SAmpling Scheme (GRASS) proposed here derives from ETR as an alternative, computationally more efficient, edge aware sampling scheme. As ETR, GRASS explores the image regions around any pixel $x$ by taking into account the magnitude of the image gradient and the spatial distance from $x$ of the sampled pixels. It also implements a stigmergy-inspired mechanism to overcome the multiple exploration of the same region.

GRASS scans the neighborhood of $x$ by means of a set of $N$ random squares with different size and orientation, and with a side containing the pixel $x$ (see Figures 1 and 2). These squares are computed as follows.

For any pixel $x, N$ pixels $y_{1}, \ldots, y_{N}$ are sampled at random from $\operatorname{Supp}(I)$ as in ETR. These $N$ pixels are chosen so that $y_{i} \neq y_{j} \neq x$ for any $i \neq j$, where $i, j=1, \ldots, N$.

For each $i$, the pair $\left(x, y_{i}\right)$ defines a square $s_{i}$ in $\operatorname{Supp}(I)$, such that:

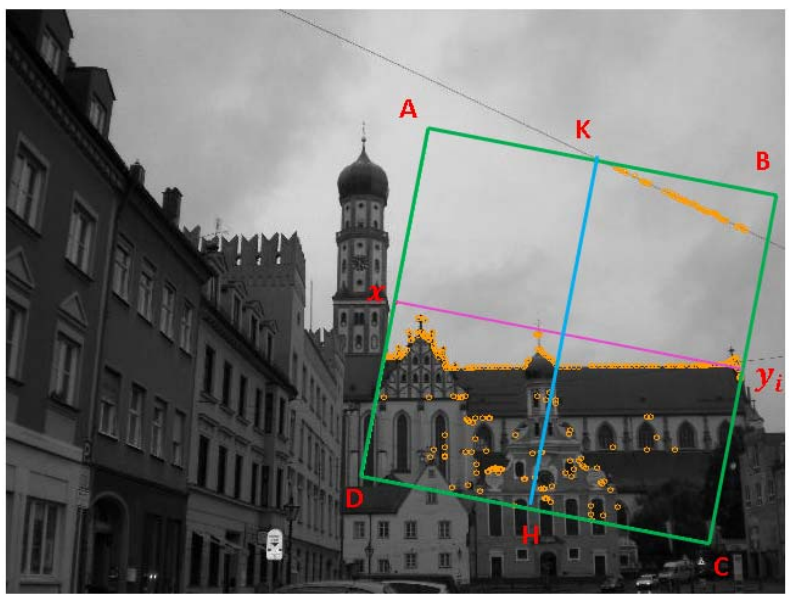

Fig. 2. An example of GRASS sampling: the square $s_{i}$ highlighted in green samples a region around the pixel $x$, that here is the center of mass of the image support. The square is defined by the segment $x-y_{i}$ (in magenta), where $y_{i}$ is a pixel randomly sampled over the image: the square is centered at $x$, the length of its sides is the length of $x-y_{i}$, i.e. $\left\|x-y_{i}\right\|$, and the side $\mathrm{AD}:=L_{i}$ containing $x$ is orthogonal to $x-y_{i}$. The square is scanned along the segments parallel to AD. HK is an example of such a segment. The orange pixels inside $s_{i}$ indicates the positions of the pixels maximizing $\phi_{x}$ over each segment scanned in $s_{i}$ (see Eq. (8)).

1) the middle point of the segment $x-y_{i}$ is the barycenter of $s_{i}$;

2) the length of the sides of $s_{i}$ is equal to the length $\left\|x-y_{i}\right\|$ of $x-y_{i}$

3) one side of $s_{i}$, let's say $L_{i}$, contains $x$ and it is orthogonal to the direction of $x-y_{i}$.

Each square $s_{i}$ defines an asymmetric neighborhood of $x$.

Figure 2 shows an example of such a square, drawn in green color on a gray level image, and defined by the barycenter of the image support (i.e. the pixel $x$ ) and by the pixel $y_{i}$. The square vertices are named $\mathrm{A}, \mathrm{B}, \mathrm{C}, \mathrm{D}$, and $\mathrm{AD}:=L_{i}$.

GRASS scans $s_{i}$ from $L_{i}$ (AD in Figure 2) to the opposite side (BC in Figure 2) along the direction parallel to that of $L_{i}$. Due to the discrete nature of the data, such a procedure is equivalent to scan each segment of $s_{i}$ orthogonal to $x-y_{i}$ (e.g. the blue segment HK in Figure 2). Let $\sigma_{1}, \ldots, \sigma_{M}$ be all these segments, where $M$ is equal to $\left\|x-y_{i}\right\|$, rounded to the closest integer.

These segments are used to select in $s_{i}$ a set of pixels which are relevant to color sensation, i.e. they are close to $x$ and they maximize the gradient magnitude over a subset of the neighborhood of $x$. Precisely, for each $j=1, \ldots, M$, GRASS selects a pixel $u_{j} \in \sigma_{j}$ that maximizes over $\sigma_{j}$ the function $\phi_{x}: s_{i} \rightarrow \mathbf{R}$ defined as:

$$
\phi_{x}(u):=\|\nabla I(u)\| d_{G}(x, u)-\Theta(u), \quad u \in s_{i}
$$

i.e.

$$
\phi_{x}\left(u_{j}\right)=\max \left\{\|\nabla I(u)\| d_{G}(x, u)-\Theta(u): u \in \sigma_{j}\right\}
$$

where

- as in ETR, $\nabla I$ indicates the gradient of $I$;

- the function $d_{G}$ is a spatial term weighting the gradient magnitude and ranging over $[0,1]$. It is 

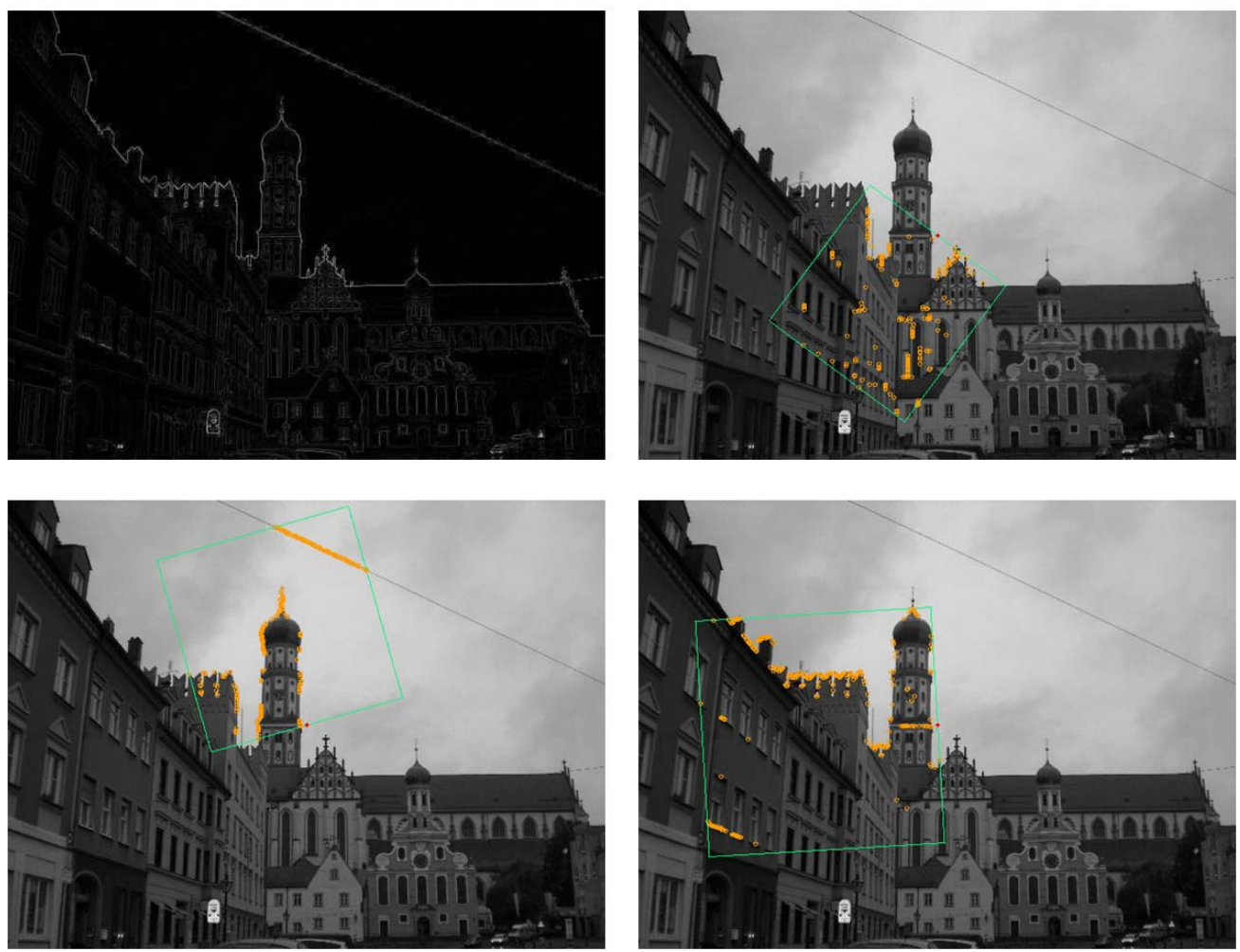

Fig. 3. The first image shows the gradient magnitude of the image in Figure 1. The other pictures show some random squares (in green) used to scan the neighborhood of the barycenter $x$ of the image support (see Figure 1). These pixels maximizes a function which is directly proportional to the gradient magnitude, while inversely proportional to their Euclidean distance from $x$.

defined as

$$
d_{G}(x, u)=1-\frac{\|x-u\|}{D}
$$

where $D$ is the length of the diagonal of $\operatorname{Supp}(I)$;

- the function $\Theta$ is a penalty term (i.e. the poison), analog to $\theta$ and $\theta_{x}$ in TR and ETR, and its values range over $\mathbf{R}$. It penalizes the multiple visit of the pixels $u_{1}, \ldots, u_{M}$ selected over the segments $\sigma_{1}, \ldots, \sigma_{M}$. This task is accomplished by initializing $\Theta$ to zero, then by increasing its values with a positive quantity $\Delta$ over the $u_{j}$ 's.

If both the pixels $u$ and $v$ of $\sigma_{j}$ satisfy Eq. (6), the algorithm selects the pixel that is the closest to $x$, in order to taking into account the influence that distance plays on the spatial color interaction [3], [16]. If $u$ and $v$ have the same distance from $x$, the algorithm chooses in $\{u, v\}$ the pixel scanned lasted. In the example reported in Figure 2, the pixels $u_{1}, \ldots, u_{M}$ maximizing $\phi_{x}$ over $\sigma_{1}, \ldots, \sigma_{M}$ are marked in orange.

Finally, GRASS scans the $3 \times 3$ windows $W_{1}, \ldots, W_{M}$ centered respectively at $u_{1}, \ldots, u_{M}$ and selects a pixel $u\left(s_{i}\right)$ such that

$$
I\left(u\left(s_{i}\right)\right)=\max _{j=1, \ldots, M} \max \left\{I(y): y \in W_{j}\right\},
$$

i.e. the pixel $u\left(s_{i}\right)$ has the maximum intensity over the set $\cup_{k=1}^{M} W_{k}$.

Figure 3 shows the gradient magnitude map (on the top, left corner) of one chromatic channel of the picture in Figure 1, some squares (in green color) sampled around the barycenter of the image support and the pixels detected by GRASS in each square (orange circles).

The chromatic component of the color sensation in $x$ is computed as

$$
S(x)=\frac{1}{N} \sum_{i=1}^{N} \frac{I(x)}{I\left(u\left(s_{i}\right)\right)},
$$

i.e. in the same way proposed by ETR.

In the current implementation of GRASS, the parameters $N$ and $\Delta$ are set up as functions of $I$, precisely:

$$
N=\frac{R+C}{2}
$$

where $R$ and $C$ denotes respectively the number of rows and columns of the input image, and

$$
\Delta=\frac{1}{2}\left[\min _{y \in \operatorname{Supp}(I)}\|\nabla I(y)\|+\max _{y \in \operatorname{Supp}(I)}\|\nabla I(y)\|\right] .
$$

For each $j=1, \ldots, M, \Theta$ is updated by the following rule:

$$
\Theta\left(u_{j}\right) \mapsto \Theta\left(u_{j}\right)+\Delta \cdot d_{G}\left(x, u_{j}\right) .
$$

We discuss the parameters set up in Section IV. Figure 4 shows some examples of square sampling with the corresponding poison map $\Theta$, iteratively updated.

The pseudo-code of GRASS procedure is sketched in Algorithm 1.

As already mentioned in Section I, the exploration mechanism of GRASS is similar to that of a flat scanner. The square 

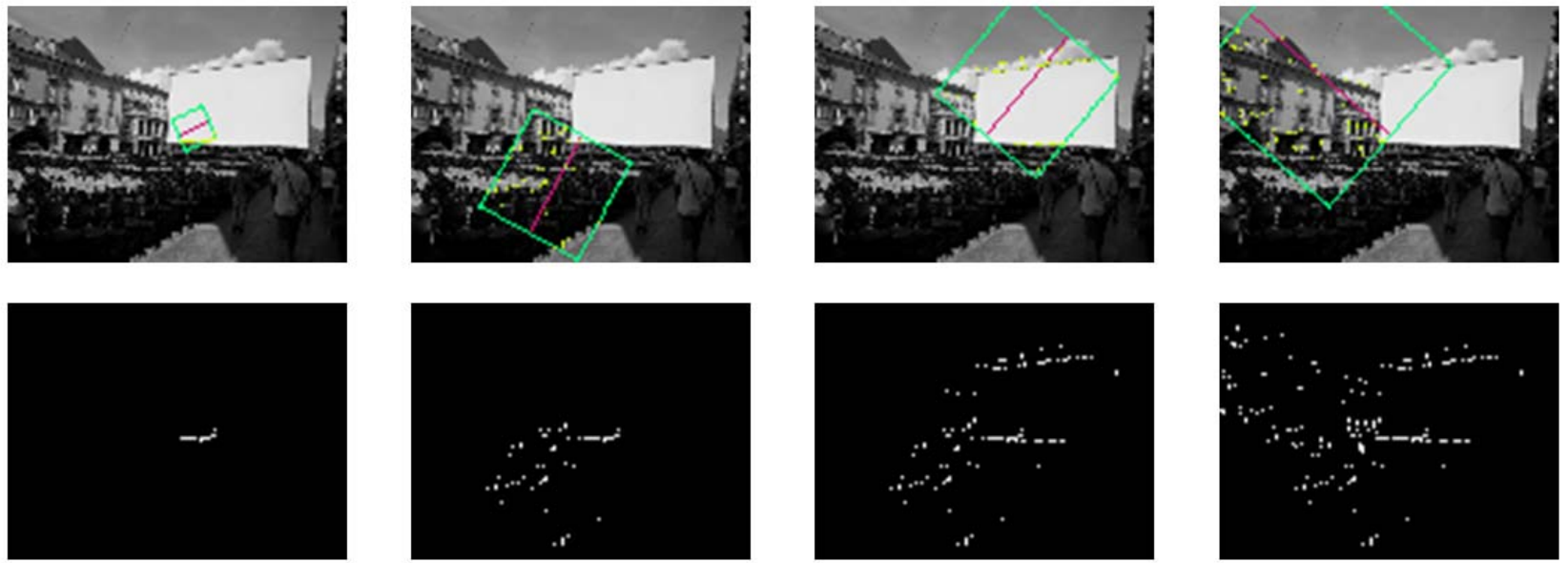

Fig. 4. Iterative image sampling by squares. From left to right: subsequent sampling squares of the center of mass of the image support (first row) and corresponding updated poison map $\Theta_{x}$ (second row).

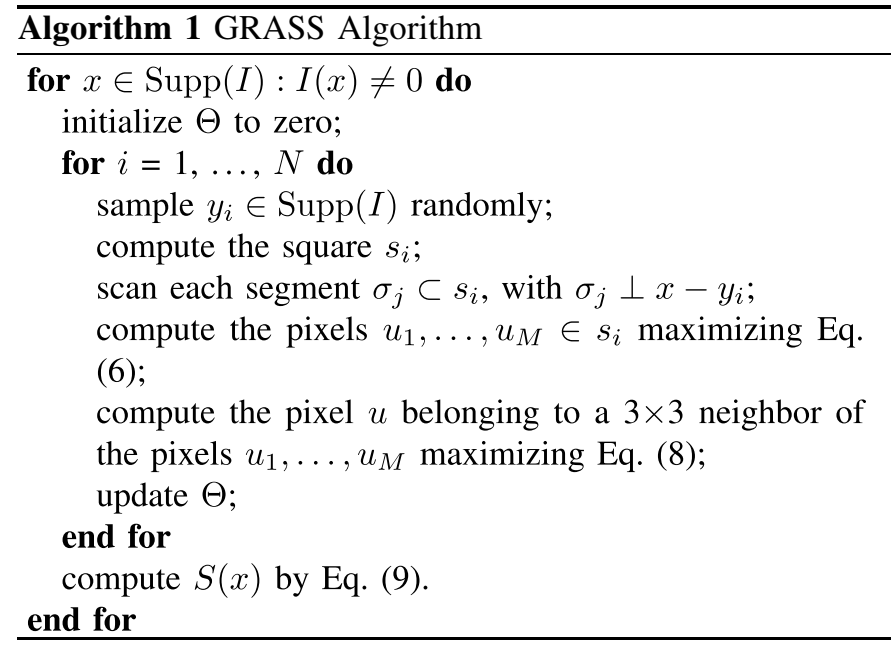

ABCD depicted in Figure 2 can be imagined as the plane of a scanner, while the segment $\mathrm{HK}$ can be seen as the scanner linear sensor moving from $\mathrm{AD}$ to $\mathrm{BC}$ (see Figure 5). The orange pixels (that are defined by Equation 6) are what the linear sensor detects during scanning the document (i.e. the square content).

Finally, we remark that the choice of squares as patches for the local exploration of the neighbor of each pixel is motivated by computational reasons: it ensures a wide image scanning with lower computational costs, compared with alternative kinds of polygons, like e.g. triangles or pentagons.

The computational complexity of GRASS is in general smaller than $\mathcal{O}\left(N|I|^{2}\right)$. In fact, a part for the pixels located on the corners, the squares used for exploring the regions around a pixel $x$ do not cover the entire image support, thus their size is smaller than $|I|$. This is an important cue: in fact, as shown in Section IV, GRASS computes sampling figures similar to those of ETR (i.e. both cover more the image edges than flat regions), but with a remarkably smaller computational burden, that makes GRASS more practicable than ETR (see Section IV for more details).
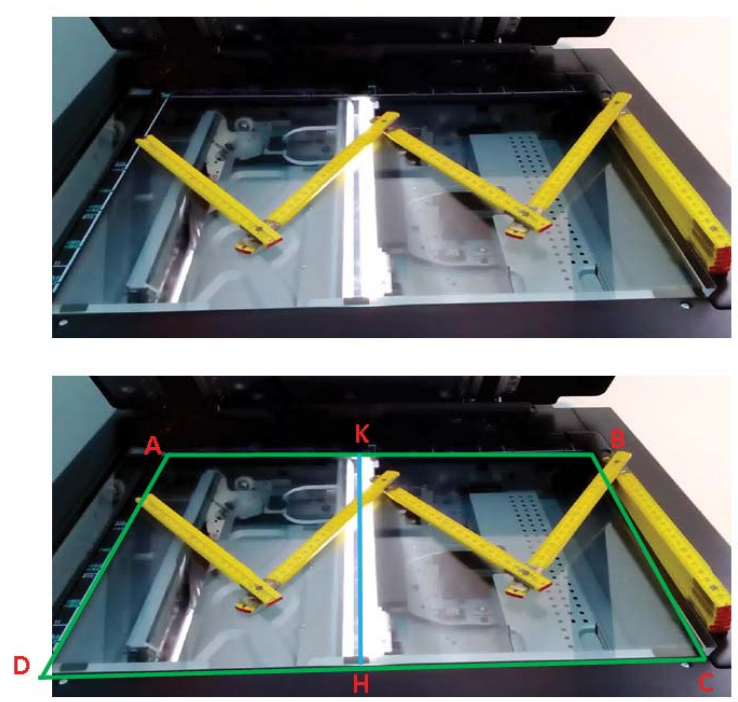

Fig. 5. Illustration of the metaphor used for explaining how GRASS selects the pixels relevant to color sensation. The sampling mechanism is similar to that of a flat scanner (on top), that explores the surface of the document to be scanned by the light emitted by the linear sensor. Each GRASS square (e.g. ABCD) can be intrepreted as the scanner plane, the image portion to be explored as the document to be scanned, and each segment scanning the square (e.g. HK) as the scanner sensor (figure on bottom). In this framework, the yellow rule on the scanner plane would like to represent an edge to be used by GRASS for estimating the color sensation. See also Figure 2.

\section{EXPERIMENTS}

Before introducing results and measures, it is important to clarify differences in Retinex tasks. As described in more details in [3] and [5], Retinex was born as a computational model of the human color sensation, but later on it has been used for much simpler tasks, like e.g. image enhancement. The use of Retinex for image enhancement does not require any particular pre- or post-filtering calibration, that on the contrary is necessary for the more complex task of human vision modeling [5]. Nevertheless, the kind of image enhancement performed by Retinex is always in the direction of the human color sensation, but to assess it quantitatively requires a careful 
procedure of input and output calibration. Thus, although without such calibrations Retinex can be seen as a qualitative (not quantitative) estimate of color sensation, in this section we prefer to refer to the output values as filtered image.

The evaluation of GRASS has been carried out by measuring its performance on visual image enhancement. In the next subsections, we present the measures used for quantifying this performance, the databases, and the results.

\section{A. Evaluation}

We assess quantitatively the GRASS performance on:

\section{i) Image enhancement.}

Retinex works as an image enhancer, i.e. the output image has equalized brightness and more visibile details than the input one. Since the color filtering depends on the pixels sampled over the image, the analysis of the image output by a Retinex algorithm allows to perform an undirected evaluation of the used sampling scheme: if no enhancement is obtained on dark images with poorly visible edges, then the sampling scheme adopted by that algorithm and/or the usage of the sampled information are probably not accurate or not appropriate for this task. In general, there are no agreed measures to assess the degree of the image enhancement [3], [22].

In this work, we are interested in measuring numerically the variations of three image features that are usually modified by Retinex algorithms: the brightness, the contrast and the dynamic intensity range. Therefore, we compare the input image and the filtered one by means of three quantitative measures $f_{0}, f_{1}, f_{2}$ explained in the following. All these measures or some of them have been already used in previous works [12], [23]-[25], for assessing the performance of Retinex approaches.

For any image $I$ with luminance $\mathcal{L}$,

- $f_{0}$ is the mean value of $\mathcal{L}$;

- $f_{1}$ is the multi-resolution contrast of $\mathcal{L}$, proposed in [26]; $f_{1}$ is obtained by averaging over multiple scaled versions of the input luminance the mean value of the local contrast of each pixel, computed over a $3 \times 3$ neighborhood;

- $f_{2}$ is the flatness of the probability density function (pdf) of the luminance introduced in [23] and given by the $L^{1}$ distance between the pdf of the luminance values and an uniform pdf.

We expect that the values of $f_{0}$ and $f_{1}$ of the filtered image are greater than those of the input image. Conversely, we expect that the flatness $f_{2}$ of the pdf of the filtered image is smaller than that one of the pdf of the input image. This is because the Retinex algorithms generally stretch the luminance value, producing a flatter pdf. Here we represent the pdf of the image luminance by a normalized histogram with 256 bins.

ii) Spatial and color sampling.

In order to investigate the mechanism of the spatial exploration and color sampling of GRASS, we measure the range of chromatic intensity and the size of the image portion effectively explored in the image enhancement tests. In particular, given an image $I$, for each pixel of $I$, we estimate the percentage of image area covered by the pixels selected by GRASS and the percentage of intensity values sampled by GRASS. These values, indicated by $f_{3}$ and $f_{4}$ are averaged over the number of image pixels, i.e. they are defined as

$$
\begin{aligned}
f_{3} & =\frac{\#\{\text { Intensity Values Sampled from } I\}}{\#\{\text { Intensity Values in } I\}} \times 100 \\
f_{4} & =\frac{\#\{\text { Pixels Sampled from Supp }(I)\}}{|I|} \times 100 .
\end{aligned}
$$

The value of $f_{3}$ indicates the percentage of different intensity values present in the image and considered in the computation of the final image; usually, a good sampling is characterized by a high value of $f_{3}$. The value of $f_{4}$ indicates the percentage of the size of the image portion explored by GRASS, averaged over the number of image pixels. If the input image contains wide flat regions, then a small value of $f_{4}$ denotes a good sampling in the overall image, with a sparse sampling of the uniform regions.

iii) Observer Judgements.

We measure the GRASS image enhancement performance also from a perceptual point of view. For this task, we considered a dataset of color images, depicting real-world scenarios. The images of this dataset have been filtered by GRASS. The original and the filtered pictures have been displayed on a black background on a monitor Samsung SyncMaster 2243SN 21.5 " to a set of 22 observers. The order of the images on the screen has been randomly choosen at the begin of the experiments and was the same for each observer. The set of observers includes people with different age, gender, and work. Observers were asked to choose the preferred image in terms of the informative content and the details.

iv) CIELab Distance.

For the pictures of COLOR-20, we also report the mean value of the CIELab distances $\triangle E$ under illuminant D65, before and after applying GRASS, averaged over the number of pictures. We remind that $\Delta E$ is the $L^{1}$ distance between theinpuit and the output image in the Lab color space. This distance is widely used to measure the perceptual differences between an image and its filtered version [17], [27], [28].

\section{B. Databases}

We report the evaluation results of GRASS on two different image databases, named TEST5GRAY and COLOR-20.

TEST5GRAY is a simple, challenging set of five grey-level images, that are identified in the next by the numbers from 1 to 5 (see Figure 6(a)) and that have been already employed in [12] for analyzing and testing ETR. The structural features of these pictures are highly significant to study, illustrate, and compare the image processing of Retinex algorithms. The usage of gray level images for evaluating Retinex algorithms is justified by the fact that the Retinex processes each chromatic channel separately, therefore image enhancement and spatial sampling can be investigated on one-channel images, allowing to better visualize the final effects and the sampling scheme. 
(a)

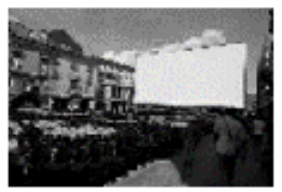

(b)

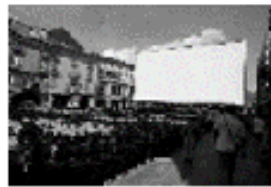

(c)

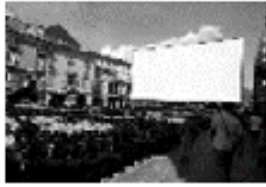

(d)

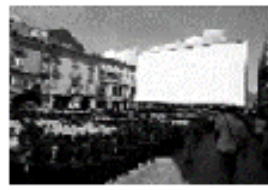

(e)

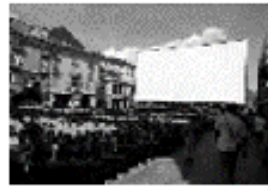

(f)

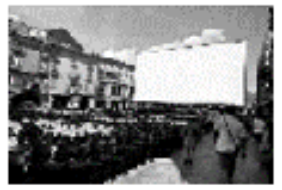

(g)

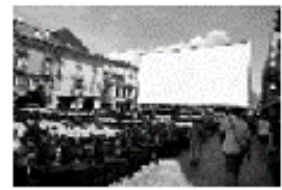

(h)

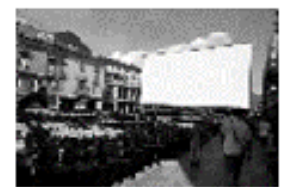

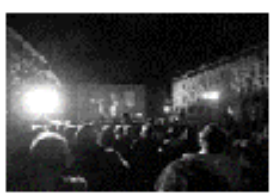
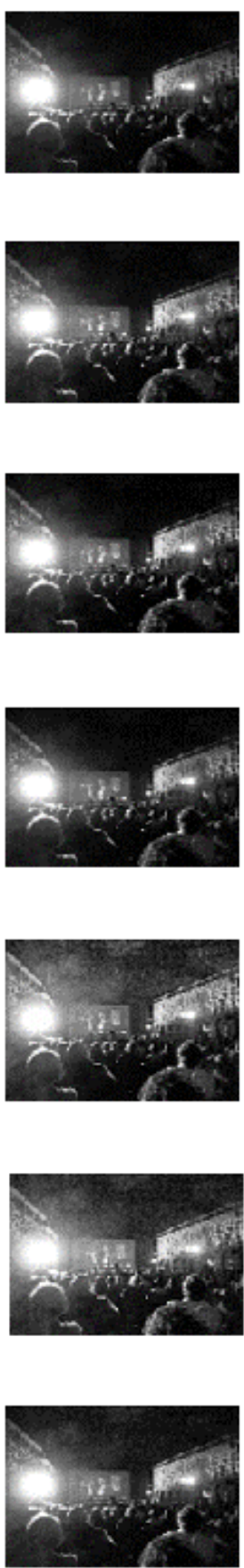
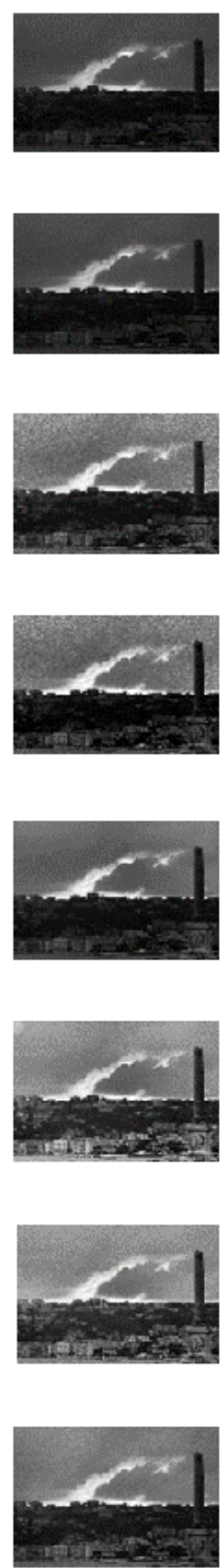
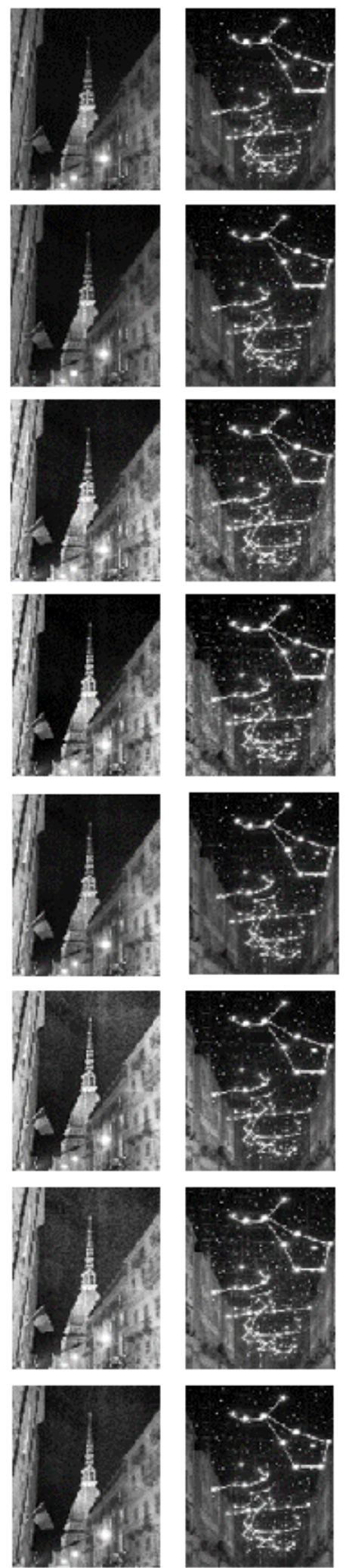

Fig. 6. Comparison among the filtering results obtained by using different Retinex algorithms: (a) no filtering (input images); (b) Scale-by-Max; (c) RSR $(\mathrm{N}$. of sprays $=10, \mathrm{~N}$. of samples per spray $=100)$; (d) STRESS $(\mathrm{N}$. of sprays $=10$, N. of samples per spray $=100$ ); (e) L-QBRIX (quantile $=0.995)$; (f) TR (parameters as in [12]); (g) ETR (parameters as in [12]); (h) GRASS ( $N$ and $\Delta$ as in Equations (10) and 12)).

Therefore we use TEST5GRAY to show and evaluate the GRASS performance on image enhancement also in comparison with TR, ETR, RSR, STRESS and L-QBRIX.
The five images of TEST5GRAY are characterized by a different spatial distribution of bright and dark regions: Image 1 contains a very large, almost clipped white patch; 
TABLE I

TeSt5GRay: Performance on Image Enhancement. (a) Mean Luminance $f_{0}$. (b) Multi-Resolution Contrast $f_{1}$. (c) Histogram Flatness $f_{2}\left[\cdot 10^{-3}\right]$

(a)

\begin{tabular}{|l|c|c|c|c|c|c|c|c|}
\hline Image & Input & Scale-by-Max & RSR & STRESS & L-QBRIX & TR & ETR & GRASS \\
\hline 1 & 91.4 & 94.7 & 102.0 & 101.4 & 101.0 & 117.0 & 118.6 & 105.5 \\
\hline 2 & 51.9 & 51.9 & 59.3 & 56.2 & 54.1 & 79.8 & 80.8 & 62.89 \\
\hline 3 & 55.4 & 55.4 & 89.9 & 81.1 & 75.7 & 110.8 & 112.3 & 90.01 \\
\hline 4 & 61.1 & 61.1 & 90.0 & 82.2 & 81.8 & 102.5 & 105.3 & 82.87 \\
\hline 5 & 52.0 & 52.0 & 70.2 & 64.2 & 54.8 & 63.5 & 71.1 & 56.51 \\
\hline
\end{tabular}

(b)

\begin{tabular}{|l|c|c|c|c|c|c|c|c|}
\hline Image & Input & Scale-by-Max & RSR & STRESS & L-QBRIX & TR & ETR & GRASS \\
\hline 1 & 22.8 & 23.7 & 25.8 & 25.8 & 25.3 & 29.3 & 29.1 & 26.6 \\
\hline 2 & 17.2 & 17.2 & 19.7 & 19.9 & 18.1 & 20.9 & 21.4 & 19.3 \\
\hline 3 & 10.7 & 10.7 & 18.0 & 18.6 & 14.2 & 19.6 & 18.3 & 15.8 \\
\hline 4 & 17.2 & 17.2 & 24.7 & 25.8 & 22.8 & 25.4 & 24.5 & 21.8 \\
\hline 5 & 19.5 & 19.5 & 23.8 & 24.8 & 20.3 & 21.8 & 22.5 & 20.5 \\
\hline
\end{tabular}

(c)

\begin{tabular}{|l|c|c|c|c|c|c|c|c|}
\hline Image & Input & Scale-by-Max & RSR & STRESS & L-QBRIX & TR & ETR & GRASS \\
\hline 1 & 3.06 & 3.05 & 2.83 & 2.87 & 3.03 & 2.12 & 2.21 & 2.59 \\
\hline 2 & 3.71 & 3.71 & 3.38 & 3.45 & 3.61 & 2.58 & 2.57 & 3.30 \\
\hline 3 & 4.85 & 4.85 & 3.07 & 3.10 & 3.92 & 2.94 & 3.10 & 3.56 \\
\hline 4 & 3.41 & 3.41 & 2.39 & 2.42 & 2.65 & 1.82 & 2.12 & 2.65 \\
\hline 5 & 4.20 & 4.20 & 3.05 & 3.11 & 3.95 & 3.20 & 3.21 & 3.86 \\
\hline
\end{tabular}

Image 2 contains two very bright, isolated bright spots; Image 3 is characterized by an elongated bright region against a dark background; Image 4 and 5 contain the same issues of the other images, but with different proportions.

The dataset COLOR-20 consists of 20 color images, depicting real-world scenarios including both indoor and outdoor environments, along with the color versions of the five images of TEST5GRAY (see Figure 9). The images of this dataset are grouped in two subsets upon their characteristics (see Figures 11(a) and 12): group 1 contains 10 images, underexposed or captured under bad illuminant conditions, and thus very dark and with poorly visible details; group 2 contains 10 images, with a good visibility of the details, composed by dark and bright regions and/or characterized by a chromatic dominant due to the light under which these pictures have been captured. Here, we use COLOR-20 for two tasks: first, for showing and evaluating the GRASS image enhancement performance on color images; second, for providing a human based evaluation of the GRASS color filtering.

\section{Results on Visual Enhancement}

Figure 6 reports the estimates of the color filtering achieved by using GRASS, TR, ETR, RSR, STRESS, QBRIX and Scale-by-Max on TEST5GRAY. We remind that Scale-by-Max is a well known limit case of global Milano Retinex [3]: in this case, the output is computed by rescaling each intensity value of the input image by the maximum channel intensity. In these experiments, the parameters $N$ and $\Delta$ of GRASS have been set up as in Equations (10) and (12), while those ETR have been fixed as in [12]; the parameters of TR have been estimated in order to have the same number of termites of ETR and a poison value similar to that of ETR; for RSR and STRESS,
TABLE II

TEST5GRAY: EVALUATION OF THE EXPLORATION SCHEME. (a) $f_{3}$. (b) $f_{4}$

(a)

\begin{tabular}{|l|c|c|c|}
\hline Image & RSR and STRESS & ETR & GRASS \\
\hline 1 & 54.70 & 99.60 & 95.99 \\
\hline 2 & 46.70 & 99.20 & 99.36 \\
\hline 3 & 38.76 & 98.82 & 99.84 \\
\hline 4 & 48.96 & 97.08 & 96.75 \\
\hline 5 & 43.68 & 92.82 & 99.71 \\
\hline
\end{tabular}

(b)

\begin{tabular}{|l|c|c|c|}
\hline Image & RSR and STRESS & ETR & GRASS \\
\hline 1 & 2.74 & 47.53 & 46.97 \\
\hline 2 & 2.74 & 42.84 & 51.57 \\
\hline 3 & 2.74 & 56.78 & 61.68 \\
\hline 4 & 2.71 & 63.62 & 51.65 \\
\hline 5 & 2.71 & 63.67 & 50.86 \\
\hline
\end{tabular}

the number of sprays and the number of samples per spray are respectively 10 and 100; finally, the quantile used in L-QBRIX is 0.995 .

As shown in Figure 6, the image enhancement produced by Scale-by-Max is negligible, while that produced by the Retinex algorithms RSR, STRESS, L-QBRIX, TR, ETR and GRASS is much more visible: the filtered images are brighter and have a higher contrast, that makes clearer the details contained in the dark areas. Table I reports the values of $f_{0}, f_{1}, f_{2}$ for these images. We observe that the results obtained by GRASS are in line with those achieved by the other approaches, i.e. GRASS works as an image enhancer.

The values of $f_{3}$ and $f_{4}$ for GRASS are reported in Table II, along with those of ETR, RSR and STRESS. On average, 

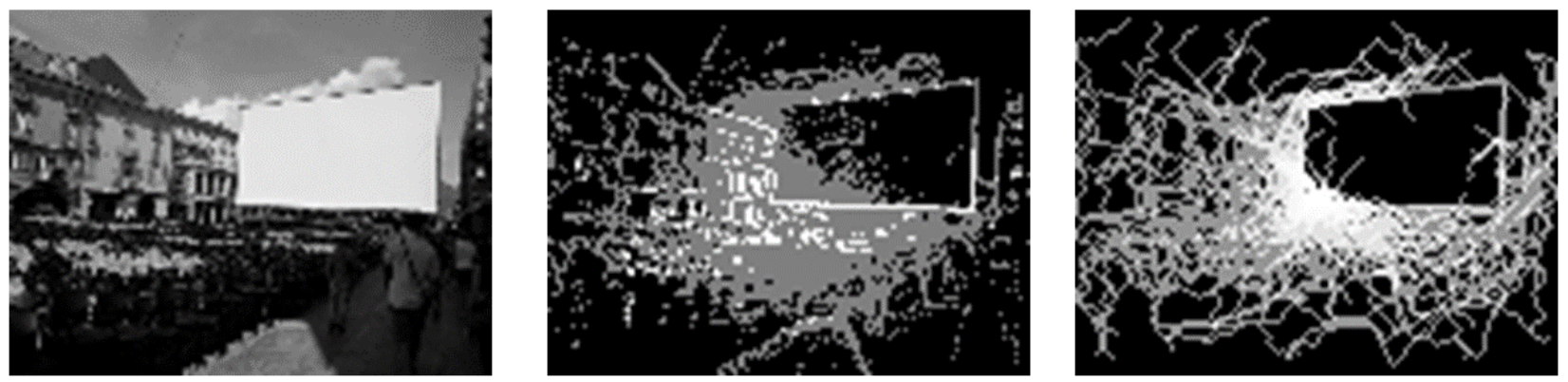

Fig. 7. A 1D image (on left) and the poison maps of GRASS (in the middle) and of ETR (on right). The poison map is computed with respect to the center of mass of the image support.

GRASS takes into account more than the $90 \%$ of the image intensity levels $\left(f_{3}\right)$, by exploring about the $50 \%$ of the area of the input image $\left(f_{4}\right)$. The value of $f_{3}$ and $f_{4}$ of RSR (and of STRESS, that adopts the RSR sampling scheme) are quite low: for the parameters used in our experiments, the size of the explored space is less than the $3 \%$ of the image size, while, for the images of TEST5GRAY, the range of the intensity levels sampled is on average less than the 55\% of the whole possible range of intensities. For Scale-by-Max and L-QBRIX, the values of $f_{3}$ and $f_{4}$ can be considered as "limit cases", because these approaches do not perform image sampling. In particular, Scale-by-Max scans the whole image support to pick up the maximum chromatic intensities, but it does not consider any spatial information. L-QBRIX also scans the whole image support and the whole range of the chromatic intensities in order to compute the spatial weighted pdf of the intensity at each pixel. For both the algorithms, $f_{3}$ and $f_{4}$ are equal to the $100 \%$. We also observe that they use a single intensity value for re-scaling the intensity of each pixel: the maximum intensity for Scale-by-Max and the intensity determined by the quantile input by the user for L-QBRIX. Regarding TR, from the mathematical formulation it follows that $f_{3}$ and $f_{4}$ are equal to or greater than those of ETR, because TR generally explores more space than ETR.

ETR and GRASS output quite similar values for $f_{3}$ and $f_{4}$. Compared to ETR, which uses the same visual cues as GRASS, GRASS has a wider spreading of the sampling, maintaining at the same time more focus on the regions with high gradient magnitude (see Figure 7), i.e. for each pixel $x$, the sampling figure at $x$ covers well the image edges and it is more dense in the region close to $x$ than far away. This is due to the local behaviour of GRASS, that maximizes the gradient magnitude weighted by spatial distance in image subset around $x$ (i.e. the squares), while ETR searches curves defined over the whole image support. In this respect the sampling figure of GRASS presents some similarities with that of RSR and STRESS, where pixel sampling is constrained by a radial density function. Consequently, as observed above, the values of $f_{0}, f_{1}, f_{2}$ are between those of the methods RSR, STRESS, L-QBRIX and those of TR and ETR.

From the computational point of view, GRASS provides execution times remarkably lower with respect to those of ETR. For instance, the current $\mathrm{C}++$ implementations of ETR and GRASS process a gray-level image with size $64 \times 64$ respectively in 100 and 10 minutes on a standard PC, Intel(R) Core(TM) i7 CPU 870 at $2.93 \mathrm{GHz}$ with operating system Linux. Despite this reduction, we are aware that the computation time of GRASS is still long. Code optimization is out of the scope of this paper, however we notice that the current implementation can be implemented on GPUs with the CUDA parallel programming model, saving one more order of magnitude in the execution time.

We also measured the performance of GRASS on visual enhancement by changing the values of the parameters $N$ and $\Delta$. We first observe that a too low value of $N$ produces noisy images, while a too high value of $N$ determines an overexploration of the image, that tends to cancel the local effects of the filtering. By using $\frac{N}{2}$ or $\frac{N}{4}$ squares, we obtain very similar results: the average luminance and the local contrast were slightly smaller, while the flatness of the luminance pdf was slightly higher than those obtained by using $N$. A low value of $\Delta$ forces the algorithm to visit multiple times the same edges, leading to a filtered image very similar to that obtained by using the global Scale-by-Max. Conversely, a very high value of $\Delta$ may produce a wide coverage of the image area, making the gradient information superfluous.

We observe that like in many Retinex implementations, also in GRASS the exploration of the image is based on random sampling. For this reason, the single pixel value may change across different runs of the algorithm. However, once the sampling parameters have been fixed, the perceptual difference among the many possible output is negligible. A detailed discussion of the relationship between the random sampling and its repeatable version can be found in [9] and [19].

Finally, we give some examples of color filtering on color pictures. Figure 8 shows the color filtering of GRASS for the color versions of the image used for explaining the GRASS sampling scheme in Figures 1,2 and 3. Figure 9 shows the results on the color version of the images of TEST5GRAY, while Figure 10 shows other examples from COLOR-20. In all these examples, the algorithm produces a brighter image, with more visible details.

Table III reports the measures evaluating the image enhancement performance of GRASS on COLOR-20. The values of $f_{i}, i=0, \ldots, 4$, have been averaged over the number of images. The measures $f_{0}, f_{1}, f_{2}$ have been computed on the 

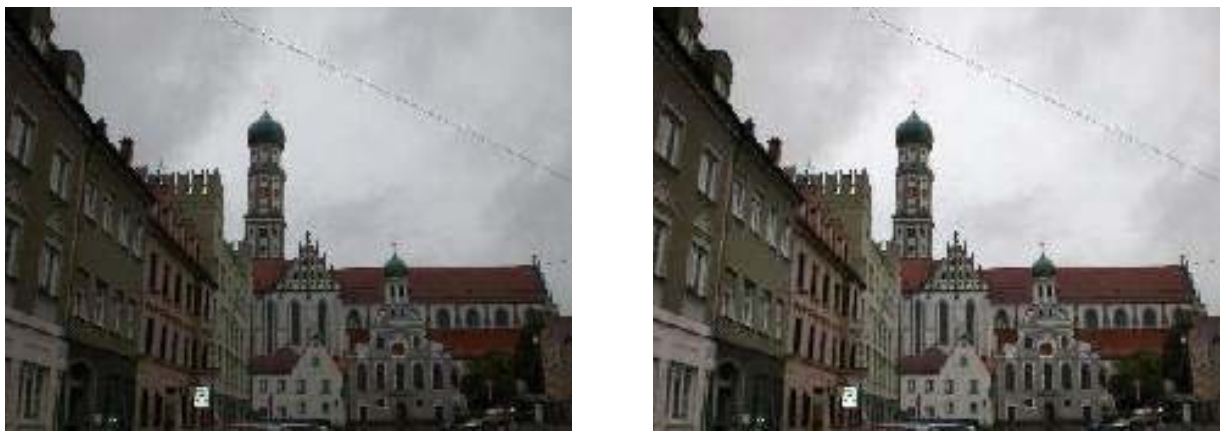

Fig. 8. Results on the image used in Figures 1,2 and 3 to explain the sampling scheme GRASS. The picture on left is the input, the picture on right is the color filtered version. The image brightness increases from 109.8 (input image) to 132.6 (filtered image).
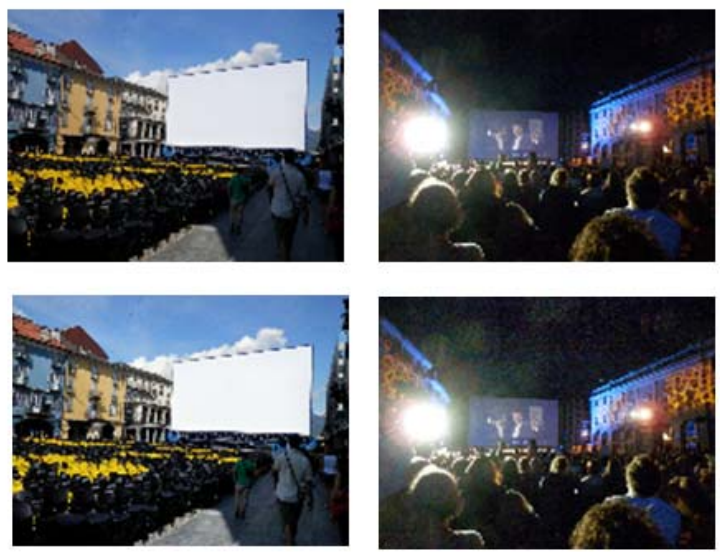
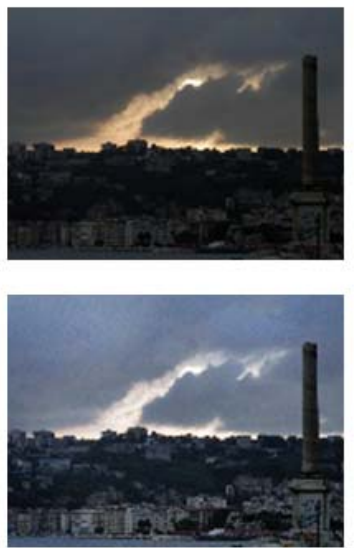
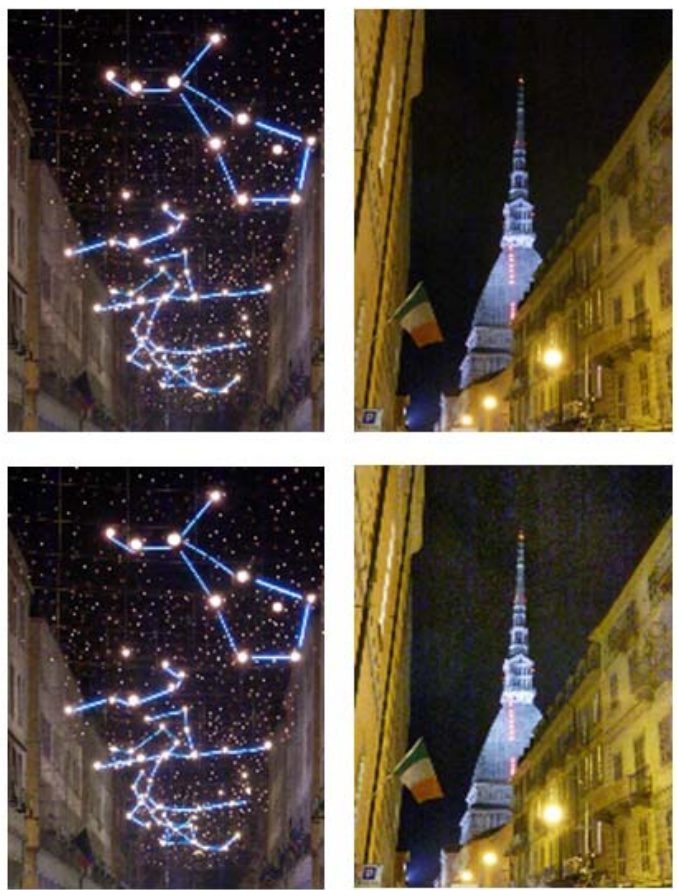

Fig. 9. COLOR-20: the color versions of the five images of TEST5GRAY (on top) and their GRASS filtering (on bottom).
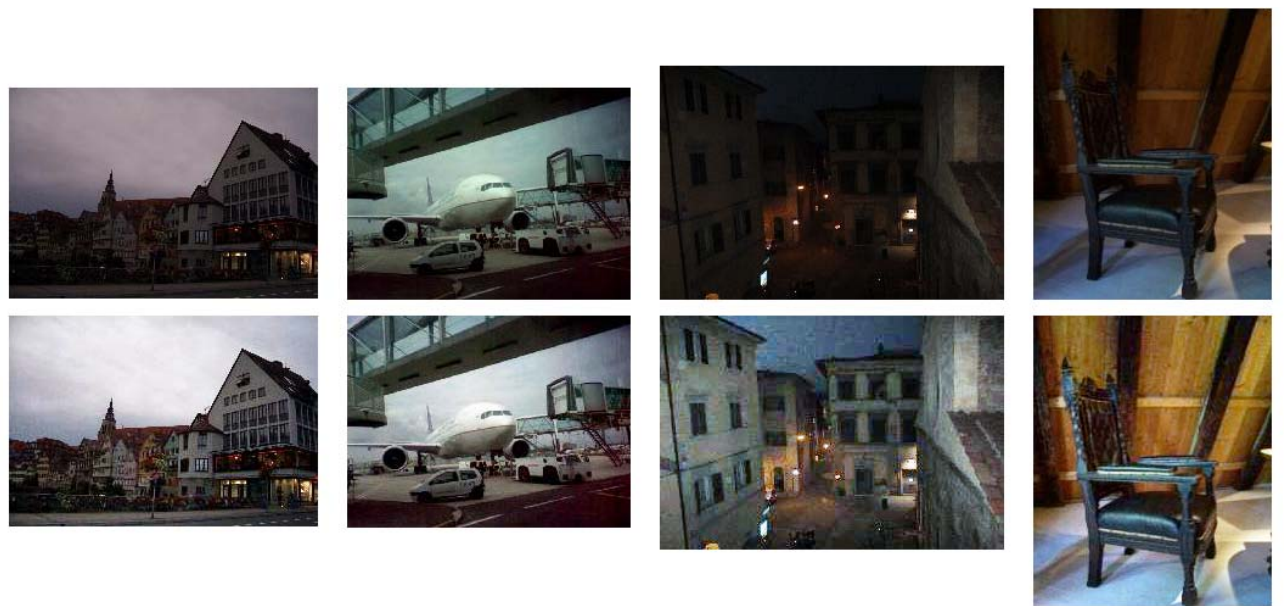
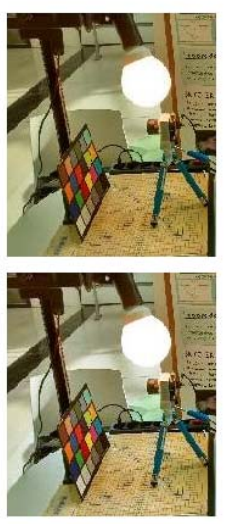

Fig. 10. COLOR-20: some images from COLOR-20 (on top) and their GRASS filtering (on bottom).

luminance of the input and output color pictures. The measures $f_{3}$ and $f_{4}$ have been computed channel by channel and then averaged over the three channels. Table IV shows these results broken down by groups. In addition, in Tables III and IV, we report the results of a comparison between the performance of GRASS and that of RSR, which, in the experiments on TEST5GRAY, provided the results most similar to those of GRASS in terms of $f_{0}, f_{1}, f_{2}$. In these tests, for RSR, 

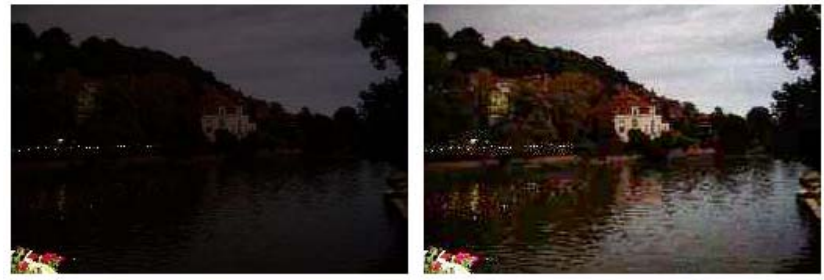

(a)
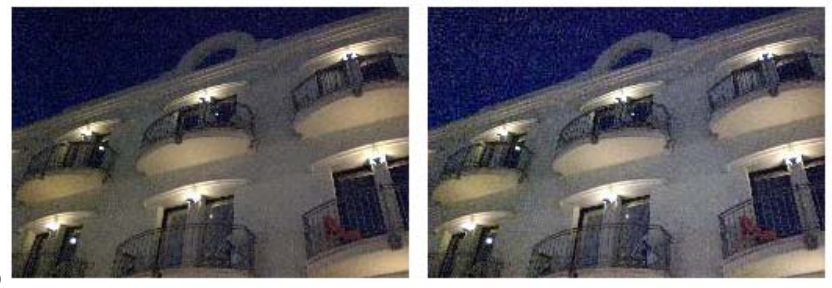

(b)
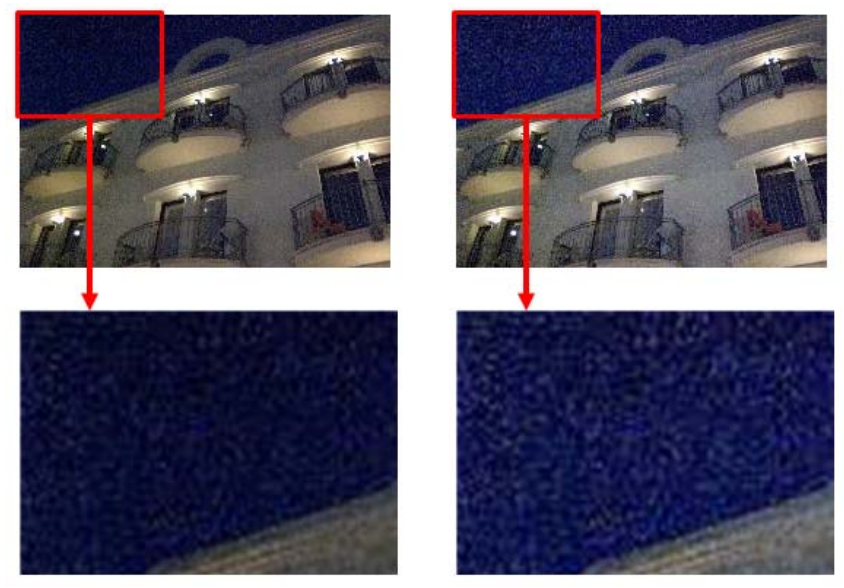

Fig. 11. COLOR-20: (a) two input images from Group 1 (top) and their GRASS filtering (bottom); for the image in the first row, the $20 \%$ of the observers expressed their preference for the GRASS output, while the $2 \%$ preferred the original image; for the image in the second row, the $6 \%$ of the observers preferred the filtered images, the 5\% had no preference, while the remaining $11 \%$ preferred the input one. This is because of the chromaric nouse, that is highlighed by the GRASS filtering (see the enlargements in (b)).

TABLE III

COLOR-20: EVALUATION OF GRASS AND RSR ON COLOR-20

\begin{tabular}{|l|c|c|c|}
\hline Measures & Input & GRASS & RSR \\
\hline$f_{0}$ & 64.72 & 92.73 & 93.07 \\
\hline$f_{1}$ & 14.40 & 19.89 & 20.47 \\
\hline$f_{2}\left[\cdot 10^{-3}\right]$ & 4.30 & 3.20 & 3.09 \\
\hline$f_{3}$ & - & 98.43 & 67.54 \\
\hline$f_{4}$ & - & 50.21 & 2.99 \\
\hline$\Delta E$ & - & 23.98 & 15.16 \\
\hline
\end{tabular}

TABLE IV

COLOR-20: EVALUATION OF GRASS AND RSR ON COLOR-20 BROKEN DOWN BY GROUPS. (a) GROUP 1. (b) GROUP 2

(a)

\begin{tabular}{|l|c|c|c|}
\hline Measures & Input & GRASS & RSR \\
\hline$f_{0}$ & 40.76 & 80.45 & 80.75 \\
\hline$f_{1}$ & 10.24 & 19.16 & 19.52 \\
\hline$f_{2}\left[\cdot 10^{-3}\right]$ & 5.03 & 3.28 & 3.16 \\
\hline$f_{3}$ & - & 99.62 & 58.47 \\
\hline$f_{4}$ & - & 49.99 & 2.81 \\
\hline$\Delta E$ & - & 26.33 & 23.73 \\
\hline
\end{tabular}

(b)

\begin{tabular}{|l|c|c|c|}
\hline Measures & Input & GRASS & RSR \\
\hline$f_{0}$ & 88.62 & 104.99 & 105.39 \\
\hline$f_{1}$ & 18.62 & 20.62 & 21.51 \\
\hline$f_{2}\left[\cdot 10^{-3}\right]$ & 3.44 & 3.08 & 3.03 \\
\hline$f_{3}$ & - & 97.12 & 76.61 \\
\hline$f_{4}$ & - & 50.30 & 3.18 \\
\hline$\Delta E$ & - & 9.21 & 6.59 \\
\hline
\end{tabular}

we considered 20 sprays per pixel and 200 samples per spray. As well as on TEST5GRAY, also on COLOR-20, RSR and GRASS exhibit a similar behaviour: the measures $f_{0}, f_{1}$, $f_{2}$ output by the two methods are very close to each other, while the size of the explored image support and the range of sampled intensities, i.e. $f_{3}$ and $f_{4}$ are remarkably different.

Finally, Table V reports the results of the perceptual tests carried out on a set of 22 observers. FP, NP and OP indicate respectively the percentage of observers preferring the filtered image (FP), that having no preference (NP), and that preferring
TABLE V

COLOR-20: Results of Perceptual Tests

\begin{tabular}{|l|c|c|c|}
\hline Image Group & FP (\%) & NP (\%) & OP (\%) \\
\hline Group 1 & 71.36 & 3.64 & 25.00 \\
\hline Group 2 & 36.82 & 21.36 & 41.82 \\
\hline All Dataset & 54.09 & 12.50 & 33.41 \\
\hline
\end{tabular}

the original image (OP). FP, NP and OP have been averaged over the number of observers and over the number of images per group. For Group 1, the filtered images have been preferred by more than the $70 \%$ of the observers, while a low percentage of people had no preferences. This means that, on average, the GRASS filtering carried out on pictured acquired with exposure problems, like those of Group 1, is considered highly performing in terms of image enhancement. The $25 \%$ of the volunteeers expressed their preference for the input images. In some cases, this preference is due to the amplifcation of the chromatic noise produced by GRASS on some images of the Group 1. In fact, the pictures of COLOR-20 have been acquired by commercial cameras with bad exposure or under low illuminant conditions in JPEG format: due to these issues, the image is affected by chromatic noise, that GRASS - as well all the algorithms of the Retinex family - tends to emphasize. Therefore, the output images may be more noisy than the original ones, causing a preference for the not filtered versions (see for instance Figure 11(b)). For Group 2, that contains images taken with a better exposure or under better light conditions, the $21 \%$ of the observers had no preferences, while the $37 \%$ of them considered augmented the content of the filtered images. The remaining $42 \%$ of the observers preferred the input images. The separation between the persons preferring the input and those preferring the output is not very evident. In general, as reported in Table IV, for the images of Group 2, GRASS produced a low increment of the contrast, so that the visibility of the details remained almost unaltered (see Figure 12 for some examples). This behavior is typical for the family of Retinex algorithms, where if the input 
(a)
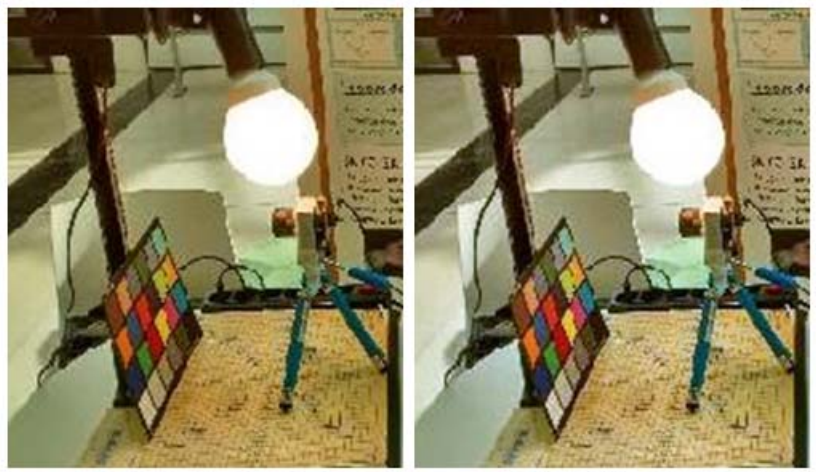

(b)

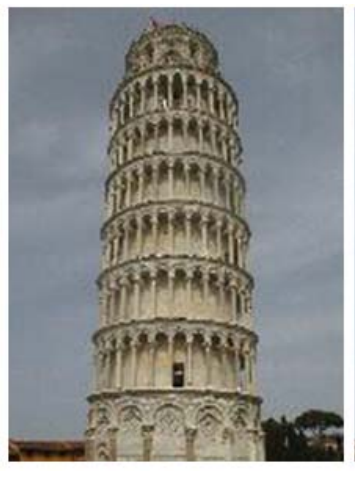

Fig. 12. COLOR-20: (a) and (b) display an image from Group2 and its GRASS filtering. For the images in (a), the 8\% of the observers expressed their preference for the GRASS output, the $8 \%$ preferred the original image, while the $6 \%$ do not have any preference; for the images in (b), the $13 \%$ of the observers preferred the filtered images, the $5 \%$ had no preference, while the remaining $4 \%$ preferred the input one.

image is well exposed and readable, they makes almost no changes. This is in line with the fact that the increment of the measures $f_{0}, f_{1}, f_{2}$ and of the perceptual CIELab distance $\Delta E$ is higher for the images in Group 1 than for those in Group 2 (see Table IV).

\section{CONCLUSIONS}

In this work, we have presented a novel spatial color sampling approach for Retinex, named GRASS. It takes inspiration from the algorithms TR and ETR, two initial implementations of Retinex proposing an image aware spatial sampling. GRASS introduces an alternative gradient sampling scheme, where the neighborhood of each pixel is explored by means of square supports with different size and orientation, and selects from each square the pixels relevant for the Retinex computation. Relevance is defined in terms of gradient magnitude and distance from the pixel for which the color sensation is computed. The pixels selected from each square can be viewed as control points of a curve scanning the square or as a $2 \mathrm{D}$ sampling, so that GRASS sampling can be classified both as a 1D and a 2D sampling approach for Retinex. In particular, as TR and ETR, the GRASS sampling figure at any pixel covers image egdes and, compared to ETR, is characterized by a wider spreading around the pixel. Due to this feature, the GRASS sampling figure presents some similarities with the sampling figures of other bi-dimensional sampling schemes, such as RSR and STRESS. The performance of GRASS as image enhancer are close to those of TR, ETR, RSR, STRESS, L-QBRIX, i.e. the color filtering of GRASS tends to increase the brightness and the details visibility of the input image. this makes GRASS an alternative image aware Retinex implementation.

From the computational point of view, the sampling scheme of GRASS has a much lower complexity than that of ETR, hower future work will be partially devoted to the optimization of the code. In addition, we plan to analyze possible other edge based visual properties that can guide the Retinex image sampling [13].

\section{ACKNOWLEDGMENTS}

The authors would like to thank Cristian Bonanomi for his help in the perceptual experiments.

\section{REFERENCES}

[1] J. Albers, Interaction of Color. New Haven, CT, USA: Yale Univ. Press, 2006.

[2] R. de Valois and K. de Valois, Spatial Vision (Oxford Psychology Series 14). Oxford, U.K.: Oxford Univ. Press, 1990.

[3] J. McCann and A. Rizzi, The Art and Science of HDR Imaging. Hoboken, NJ, USA: Wiley, 2011.

[4] E. H. Land and J. J. McCann, "Lightness and Retinex theory," J. Opt. Soc. Amer, vol. 61, no. 1, pp. 1-11, 1971.

[5] A. Rizzi and J. J. McCann, "On the behavior of spatial models of color," Proc. SPIE, vol. 6493, pp. 649302-1-649302-14, Jan. 2007.

[6] A. Rizzi, J.J, McCann, M. Bertalmio, and G. Gianini Eds, "Special issue on Retinex at 50," J. Electron. Imag., vol. 26, no. 3, 2017

[7] E. Provenzi, L. De Carli, A. Rizzi, and D. Marini, "Mathematical definition and analysis of the Retinex algorithm," J. Opt. Soc. Amer. A, Opt. Image Sci., vol. 22, no. 12, pp. 2613-2621, 2005.

[8] R. Montagna and G. D. Finlayson, "Constrained pseudo-Brownian motion and its application to image enhancement," J. Opt. Soc. Amer. A, Opt. Image Sci., vol. 28, no. 8, pp. 1677-1688, Aug. 2011.

[9] G. Gianini, A. Rizzi, and E. Damiani, "A Retinex model based on absorbing Markov chains," Inf. Sci., vol. 327, pp. 149-174, Jan. 2016

[10] A. Rizzi, "Designator Retinex, Milano Retinex and the locality issue," Electron. Imag., vol. 2016, no. 6, pp. 1-5, 2016.

[11] G. Simone, G. Audino, I. Farup, F. Albregtsen, and A. Rizzi, "Termite Retinex: A new implementation based on a colony of intelligent agents," J. Electron. Imag., vol. 23, no. 1, p. 013006-1 - 013006-13, 2014.

[12] M. Lecca, A. Rizzi, and G. Gianini, "Energy-driven path search for Termite Retinex," J. Opt. Soc. Amer. A, Opt. Image Sci., vol. 33, no. 1, pp. 31-39, 2016.

[13] N. D. Daw, "Why after-images are not seen in normal circumstances," Nature, vol. 196, pp. 1143-1145, Dec. 1962.

[14] J. H. Elder and A. J. Sachs, "Psychophysical receptive fields of edge detection mechanisms," Vis. Res., vol. 44, no. 8, pp. 795-813, 2004. [Online]. Available: http://www.sciencedirect.com/science/article/pii/ S0042698903007533

[15] K. Moutoussis, "The physiology and psychophysics of the color-form relationship: A review," Frontiers Psychol., vol. 6, p. 1407, Nov. 2015.

[16] O. Creutzfeldt, B. Lange-Malecki, and K. Wortmann, "Darkness induction, Retinex and cooperative mechanisms in vision," Experim. Brain Res., vol. 67, no. 2, pp. 270-283, 1987.

[17] E. Provenzi, M. Fierro, A. Rizzi, L. De Carli, D. Gadia, and D. Marini, "Random spray Retinex: A new Retinex implementation to investigate the local properties of the model," IEEE Trans. Image Process., vol. 16, no. 1, pp. 162-171, Jan. 2007. [Online]. Available: http://dx.doi.org/10.1109/TIP.2006.884946

[18] O. Kolås, I. Farup, and A. Rizzi, "Spatio-temporal Retinexinspired envelope with stochastic sampling: A framework for spatial color algorithms," J. Imag. Sci. Technol., vol. 55, no. 4, pp. 40503-1-40503-10, 2011.

[19] G. Gianini, A. Manenti, and A. Rizzi, "QBRIX: A quantile-based approach to Retinex," J. Opt. Soc. Amer. A, Opt. Image Sci., vol. 31, no. 12, pp. 2663-2673, Dec. 2014. [Online]. Available: http://josaa. osa.org/abstract.cfm?URI=josaa-31-12-2663 
[20] A. B. M. T. Islam and I. Farup, "Enhancing the output of spatial color algorithms," in Proc. 2nd Eur. Workshop Vis. Inf. Process. (EUVIP), Jul. 2010, pp. 7-12.

[21] A. B. M. T. Islam and I. Farup, "Spatio-temporal colour correction of strongly degraded movies," Proc. SPIE, vol. 7866, pp. 78660Z-1-78660Z-15, Jan. 2011.

[22] M. Pedersen and J. Y. Hardeberg, "Full-reference image quality metrics: Classification and evaluation," Found. Trends Comput. Graph. Vis., vol. 7, no. 1, pp. 1-80, 2012.

[23] A. Rizzi, C. Gatta, and D. Marini, "A new algorithm for unsupervised global and local color correction," Pattern Recognit. Lett., vol. 24, no. 11, pp. 1663-1677, Jul. 2003.

[24] M. Lecca and A. Rizzi, "Tuning the locality of filtering with a spatially weighted implementation of Random Spray Retinex," J. Opt. Soc. Amer. A, Opt. Image Sci., vol. 32, no. 10, pp. 1876-1887, 2015.

[25] N. Banić and S. Lončarić, "Smart light random memory sprays Retinex: A fast Retinex implementation for high-quality brightness adjustment and color correction," J. Opt. Soc. Amer. A, Opt. Image Sci., vol. 32, no. 11 , pp. 2136-2147, 2015.

[26] A. Rizzi, T. Algeri, G. Medeghini, and D. Marini, "A proposal for contrast measure in digital images," in Proc. 2nd Eur. Conf. Color Graph., Imag., Vis. (CGIV), 6th Int. Symp. Multispectral Color Sci. Aachen, Germany, 2004, pp. 187-192.

[27] C. Oleari, Standard Colorimetry: Definitions, Algorithms and Software. Hoboken, NJ, USA: Wiley, 2016.

[28] E. Provenzi, C. Gatta, M. Fierro, and A. Rizzi, "A spatially variant whitepatch and gray-world method for color image enhancement driven by local contrast," IEEE Trans. Pattern Anal. Mach. Intell., vol. 30, no. 10, pp. 1757-1770, Oct. 2008 .

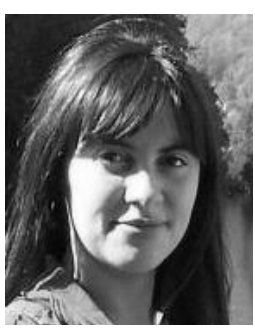

Michela Lecca received the degree in mathematics from the Università degli Studi di Trento, Italy, in 2002. In 2002, she joined the Research Unit Technologies of Vision, Fondazione Bruno Kessler, Italy, where she has a permanent position. Her current research interests include machine vision, color image processing, object recognition, image retrieval, semantic image labeling, and low-level image processing for embedded systems. She is a member of the International Association for Pattern Recognition IAPR-GIRPR and the Gruppo Italiano del Colore-Associazione Italiana Colore.

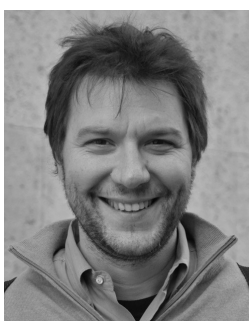

Alessandro Rizzi is the Head of the MIPS Laboratory with the Department of Computer Science. He is also Full Professor of Fundamentals of Digital Imaging and Colorimetry with the Department of Computer Science, Università degli Studi di Milano. He has authored over 300 scientific works. His research interests include digital imaging with a particular interest on color, visualization, photography, HDR and on the perceptual issues related to digital imaging, interfaces and lighting. He has been one of the founders of the Italian Color Group, Secretary of CIE Division 8, IS\&T Fellow, and the Vice President. In 2015, he was a recipient of the Davies Medal from the Royal Photographic Society. $\mathrm{He}$ is the Co-Chair at the IS\&T Conference Color Imaging: Displaying, Processing, Hardcopy and Applications, a Topical Editor of the Applied Color Science of the Journal of Optical Society of America A, an Associate Editor of the Journal of Electronic Imaging, and a member of several program committees of conferences related to color and digital imaging.

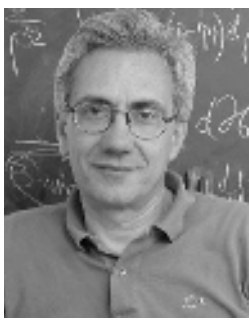

Raul Paolo Serapioni received the degree in mathematics from the Università degli Studi di Milano, Italy, and the degree from the University of Minnesota, Minneapolis, MN, USA. He was a Full Professor of Mathematical Analysis with the Politecnico di Milano, from 1990 to 2001, and since 2001, he has been with the Università degli Studi di Trento, where he was also the Head of the Department of Mathematics from 2005 to 2012. His current research interests include the regularity of solutions of degenerate elliptic and parabolic equations, geometric measure theory, Carnot-Carathodory spaces, and geometric measure theory in Carnot-Carathodory spaces. 